\title{
Prognostic Factors for Absence Epilepsy in Childhood
}

\author{
So Young Kang, MD, Chung Mo Koo, MD, Se Hee Kim, MD, Heung Dong Kim, MD, Joon Soo Lee, MD, \\ Hoon-Chul Kang, MD
}

Department of Pediatrics, Severance Children's Hospital, Yonsei University College of Medicine, Seoul, Korea

Received: August 7, 2019

Revised: September 12, 2019

Accepted: September 16, 2019

Corresponding authors:

Hoon-Chul Kang, MD

Department of Pediatrics, Severance

Children's Hospital, Yonsei

University College of Medicine,

50-1 Yonsei-ro, Seodaemun-gu,

Seoul 03722, Korea

Tel: +82-2-2228-2050

Fax: +82-2-393-9118

E-mail: hipo0207@yuhs.ac

Chung Mo Koo, MD

Department of Pediatrics, Severance

Children's Hospital, Yonsei

University College of Medicine,

50-1 Yonsei-ro, Seodaemun-gu,

Seoul 03722, Korea

Tel: +82-2-2228-2050

Fax: +82-2-393-9118

E-mail: CM0904@yuhs.ac
Purpose: Childhood absence epilepsy (CAE) is a common form of idiopathic generalized epilepsy with onset middle childhood and has typically a good prognosis, but remission rates vary. We aimed to analyze unfavorable prognostic factors in children initially diagnosed with CAE.

Methods: We retrospectively reviewed 48 patients under 13 years of age who were diagnosed with CAE at the Severance Children's Hospital, Seoul, Korea. We analyzed clinical information including comorbidity through neuropsychological test.

Results: Thirteen of the 48 patients (27\%) showed an unfavorable prognosis, with clinical seizures or seizure waves on electroencephalogram persistent even after 12 months of anticonvulsant therapy. The mean age at absence seizure onset was $6.51 \pm 2.36$ years. The most commonly used antiepileptic drug (AED) was ethosuximide, and the median duration of initial AEDs was $25.63 \pm 24.41$ months. The presence of comorbidity and clinical absence seizures after 6 months of AEDs correlated with an unfavorable prognosis. Motor seizures were the most unfavorable prognostic factor during follow-up.

Conclusion: This study shows that clinical absence seizures after 6 months of AED, comorbidity, and motor seizure are the most important predictive factors of an unfavorable prognosis for absence epilepsy in childhood. This study suggests that when these factors are observed, early intervention needs to be considered.

Keywords: Epilepsy, absence; Comorbidity; Prognosis

\section{Introduction}

Childhood absence epilepsy (CAE) is a common form of idiopathic generalized epilepsy with onset in middle childhood [1-3]. The definition of CAE is based on the frequency of absences or patterns of recurrence, and on age of seizure onset [4]. The onset age of CAE is usually between 4 and 10 years, and it is characterized by frequent and brief typical absences with abrupt impairments of consciousness. Typically, CAE leads to ictal discharges of generalized high-amplitude spikes and slow complexes on electro- encephalogram (EEG) recordings, rhythmic at $3 \mathrm{~Hz}$ with normal or mildly abnormal background activity [4-6]. Occipital intermittent rhythmic delta activity (OIRDA) on EEG is seen in one-third of children with CAE. Occasionally, children with absence epilepsy also suffer motor seizures [4]. In most patients, seizures are provoked by hyperventilation [6].

CAE is typically pharmaco-responsive, and usually treated with antiepileptic drugs (AEDs) such as ethosuximide (ETX), valproic acid (VPA), or lamotrigine (LTG) [1,2,7-9]. As the prognosis is related to various aspects of remission rates $[2,3,7-9]$. Here, we sta-

Copyright(C) 2019 Korean Child Neurology Society

This is an Open Access article distributed under the terms of the Creative Commons Attribution Non-Commercial License (http://creativecommons.org/licenses/by-nc/4.0/) which permits unrestricted non-commercial use, distribution, and reproduction in any medium, provided the original work is properly cited. 
tistically studied the prognostic factors for absence epilepsy in childhood.

\section{Materials and Methods}

\section{Patient information}

Patients who were diagnosed and treated with typical CAE at the first onset at the Severance Children's Hospital, Seoul, Korea between June 2009 and June 2017 were retrospectively reviewed. Typical CAE was defined as patients with pyknoleptic absence seizure and 3 to $4 \mathrm{~Hz}$ rhythmic generalized spike-and-wave (GSW) on EEG with normal development. Of the 78 newly diagnosed patients, 48 patients, underwent follow-up EEG at 6 and 12 months, and had started their first AEDs treatment at our hospital, were included. Patients initially diagnosed with CAE were involved in this study, but a percentage of the patients progressed to juvenile myoclonic epilepsy (JME) or juvenile absence epilepsy (JAE).

We analyzed clinical information including sex, age at absence seizure onset, EEG patterns, type and duration of AEDs, hyperventilation provocation test, whether motor seizures occurred during follow-up, and comorbidities. We also evaluated the effectiveness of AEDs at 6 and 12 months, and reviewed EEG pattern at 6 and 12 months under AEDs treatment. In this study, comorbidities analyzed through neuropsychological test including Korean Wechsler Intelligence Scale for Children-III and Korean Attention Deficit Hyperactivity Disorder (ADHD) Rating Scale. We defined a favorable prognosis as no clinical seizures and no seizure waves on EEG after the 12 months of AEDs. We defined an unfavorable prognosis as the presence of clinical or EEG seizures even 12 months after the onset of AEDs.

This study was approved by the Institutional Review Board of Severance Hospital (4-2016-0080). Informed consent was waived due to the retrospective nature of the study.

\section{Statistical analysis}

Data processing and analysis were performed with SPSS version 23.0 (IBM Co., Armonk, NY, USA). We used independent-sample t-tests, Fisher's exact test, and Pearson's chi-square test to compare variables and their relationships with prognosis. Multivariate logistic regression models with forward stepwise conditional selection of variables were used to confirm the correlation between each factor and a poor prognosis. A $P$ value $<0.05$ were considered significant.

\section{Results}

Table 1 summarizes the main characteristics of two groups, favorable and unfavorable prognosis. The favorable prognosis was de- fined as no clinical seizures and no seizure waves on EEG after the 12 months of AEDs. The unfavorable prognosis was defined as persistence of clinical seizures or seizure waves on EEG even after the 12 months of AEDs. The Thirteen of the 48 patients (27\%), showed an unfavorable prognosis. Twenty-nine patients were female, 19 were male, and the total female ratio was thus $60.4 \%$. Mean age at absence seizure onset was $6.51 \pm 2.36$ years (range, 3.0 to 12.5 ), the mean age of the patients with a favorable prognosis was $6.42 \pm 2.20$ years (range, 3.33 to 12.5 ), and the mean age of the patients with an unfavorable prognosis were $6.75 \pm 2.86$ years (range, 3.0 to 1.92 ), with no significant differences between the groups. In the EEG patterns, 3 to $4 \mathrm{~Hz}$ GSW complexes were observed in 35 patients (72.9\%) on normal background, and OIRDA was observed in eight patients (16.7\%). The median duration of initial AED was 25.63 \pm 24.41 , $23.63 \pm 13.83$ months in the group with a good prognosis and $31.00 \pm 41.83$ months in the group with a poor prognosis, respectively. The hyperventilation provocation test was negative in 13 patients $(27.1 \%)$.

In 19 patients (39.6\%), follow-up EEG was normalized after 6 months of AED therapy, while GSW complexes remained visible in 29 patients $(60.4 \%)$. In 33 patients $(68.8 \%)$, clinical seizures improved after 6 months of AED therapy, while clinical seizures were still observed in 15 patients (31.2\%). Of the 33 patients who had no clinical seizures after 6 months, only 19 patients also showed improved EEG seizures. Ten patients (20.8\%) had at least one motor seizure during the follow-up, and 10 patients (20.8\%) had comorbidities. Three of these had ADHD according to Diagnostic and Statistical Manual of Mental Disorders (DSM)-IV diagnostic criteria, while four patients only had attention deficits. One patient had a tic disorder, one patient had claustrophobia, and two patients had febrile motor seizures. Six of the 10 patients with comorbidities had a poor prognosis.

Three AEDs, ETX, VPA, and LTG were used in this study. The most commonly used AED was ETX ( 7.5 to $25 \mathrm{mg} / \mathrm{kg} /$ day), which was taken by 26 patients (54\%). VPA ( 5 to $30 \mathrm{mg} / \mathrm{kg} /$ day) was the second most common AED, and LTG (0.2 to 4.5 $\mathrm{mg} / \mathrm{kg} /$ day) was the third. Thirty-two of patients $(66.6 \%)$ used a second AED including VPA, ETX, and LTG due to uncontrolled seizures on the first AED or the side effects of the first AED. When ETX was used as the 1st AED, VPA was used as the 2nd AED in 13 patients (75\%), and LTG was used as the 2 nd AED in four patients (25\%). When VPA was used as the 1 st AED, ETX was used as the 2nd AED in seven patients (64\%), and LTG was used as the 2 nd AED in four patients (36\%). When LTG was used as the 1st AED, VPA and ETX were selected as the 2nd AED in the same ratio. 
Table 1. Characteristics of patients with childhood absence epilepsy according to prognosis

\begin{tabular}{|c|c|c|c|c|}
\hline Characteristic & Favorable prognosis $(n=35)$ & Unfavorable prognosis $(n=13)$ & Total $(n=48)$ & $P$ value \\
\hline Female (\%) & $20(57.1)$ & $9(69.2)$ & $29(60.4)$ & 0.522 \\
\hline Onset age (years old) & $6.42 \pm 2.20(3.33-12.5)$ & $6.75 \pm 2.86(3.00-11.92)$ & $6.51 \pm 2.36(3.00-12.5)$ & 0.714 \\
\hline EEG GSW duration (sec) & & & 39 (81.3) & 1.000 \\
\hline$<3$ & $7(22.6)$ & $1(12.5)$ & $8(20.5)$ & \\
\hline$\geq 3$ & $24(77.4)$ & 7 (87.5) & $31(79.5)$ & \\
\hline EEG patterns & & & & 0.030 \\
\hline GSW with normal background & $29(82.9)$ & $6(46.2)$ & $35(72.9)$ & \\
\hline OIRDA & $4(11.4)$ & $4(30.8)$ & $8(16.7)$ & \\
\hline Multifocal sharp & $2(5.7)$ & $3(23.1)$ & $5(10.4)$ & \\
\hline \multicolumn{5}{|l|}{ 1st AED } \\
\hline Ethosuximide & $20(57.1)$ & $6(46.2)$ & $26(54.2)$ & \\
\hline Valproic acid & $12(34.3)$ & $4(30.8)$ & $16(33.3)$ & \\
\hline Lamotrigine & $3(8.6)$ & $3(23.1)$ & $6(12.5)$ & \\
\hline 1st medication duration (mo) & $23.63 \pm 13.83(2-72)$ & $31.00 \pm 41.83(2-138)$ & $25.63 \pm 24.41(2-138)$ & 0.544 \\
\hline $\mathrm{HV}$ provocation-negative & $8(22.9)$ & $5(38.5)$ & $13(27.1)$ & 0.298 \\
\hline EEG at $6 \mathrm{mo} F U$ & & & & 0.049 \\
\hline Normalization & $17(48.6)$ & $2(15.4)$ & 19 (39.6) & \\
\hline GSW & $18(51.4)$ & $11(84.6)$ & $29(60.4)$ & \\
\hline Clinical absence seizures at 6 mo FU & & & & 0.012 \\
\hline Negative & $28(80.0)$ & $5(38.5)$ & $33(68.8)$ & \\
\hline Positive & $7(20.0)$ & $8(61.5)$ & $15(31.2)$ & \\
\hline Motor seizures & $1(2.9)$ & $9(69.2)$ & $10(20.8)$ & 0.000 \\
\hline Comorbidities & $4(11.4)$ & $6(46.2)$ & $10(20.8)$ & 0.160 \\
\hline
\end{tabular}

Values are presented as number (\%) or mean \pm standard deviation (range).

EEG, electroencephalogram; GSW, generalized spike-and-wave complexes; OIRDA, occipital intermittent rhythmic delta activity; AED, antiepileptic drug; HV, hyperventilation; FU, follow-up.

Univariate analysis showed that a clinical seizure even after the first 6 months of AED treatment, at least one motor seizure, and comorbidities, correlated with an unfavorable prognosis (Fig. 1A). The most significant unfavorable prognostic factor was a motor seizure at least once. There was a tendency towards an unfavorable prognosis with the age at absence seizure onset increasing (odds ratio $[\mathrm{OR}], 1.060)$, which did however not reach significance $(P=0.668)$. Although not statistically significant, the prognosis was worse when seizures were not induced by a hyperventilation test (OR, 2.109). The prognosis was poor when GSW were still visible on EEG after 6 months of AED therapy (OR, 5.194), which did however not reach significance $(P=0.050)$. All results are shown in Fig. 1.

The independent variables that were significant in univariate analysis were tested using a multivariate logistic regression analysis with a forward stepwise conditional selection method. Motor seizures ranked first in the factor hierarchy of unfavorable prognosis (OR, 105.825), followed by comorbidities (OR, 14.154) (Fig. 1B). Clinical absence seizures 6 months after AEDs did not reach significance in the multivariate analysis $(P=0.082)$.

\section{Discussion}

The purpose of our study was to statistically analyze prognostic factors in children with absence epilepsy, confirming the prognostic factors identified in previous studies, and determining new predictors. Among the patients diagnosed as CAE initially, the unfavorable prognostic factors were moter seizure, comorbidity, and clinical absence seizure 6months after the start the AEDs. Because the prognosis may vary according to patient selection, patients were selected according to the International League Against Epilepsy (ILAE) criteria for JAE, JME, and CAE. CAE differs from $\mathrm{JAE}$ in that the onset age is lower and absence seizures occur more frequently $[1,8]$. JAE presents in late childhood or adolescence with less frequent typical absences and generalized tonic-clonic seizures (GTCs). JME usually presents in adolescence with prominent myoclonic jerks that characteristically occur in the morning, and GTCs [8]. The overall prognosis of CAE is favorable but remission rates vary $[2,3,7-9]$. Previously known favorable prognostic factors include a shorter interval to loss of $3-\mathrm{Hz}$ spike-and-wave complexes, the presence of OIRDA on EEG, and a prompt re- 


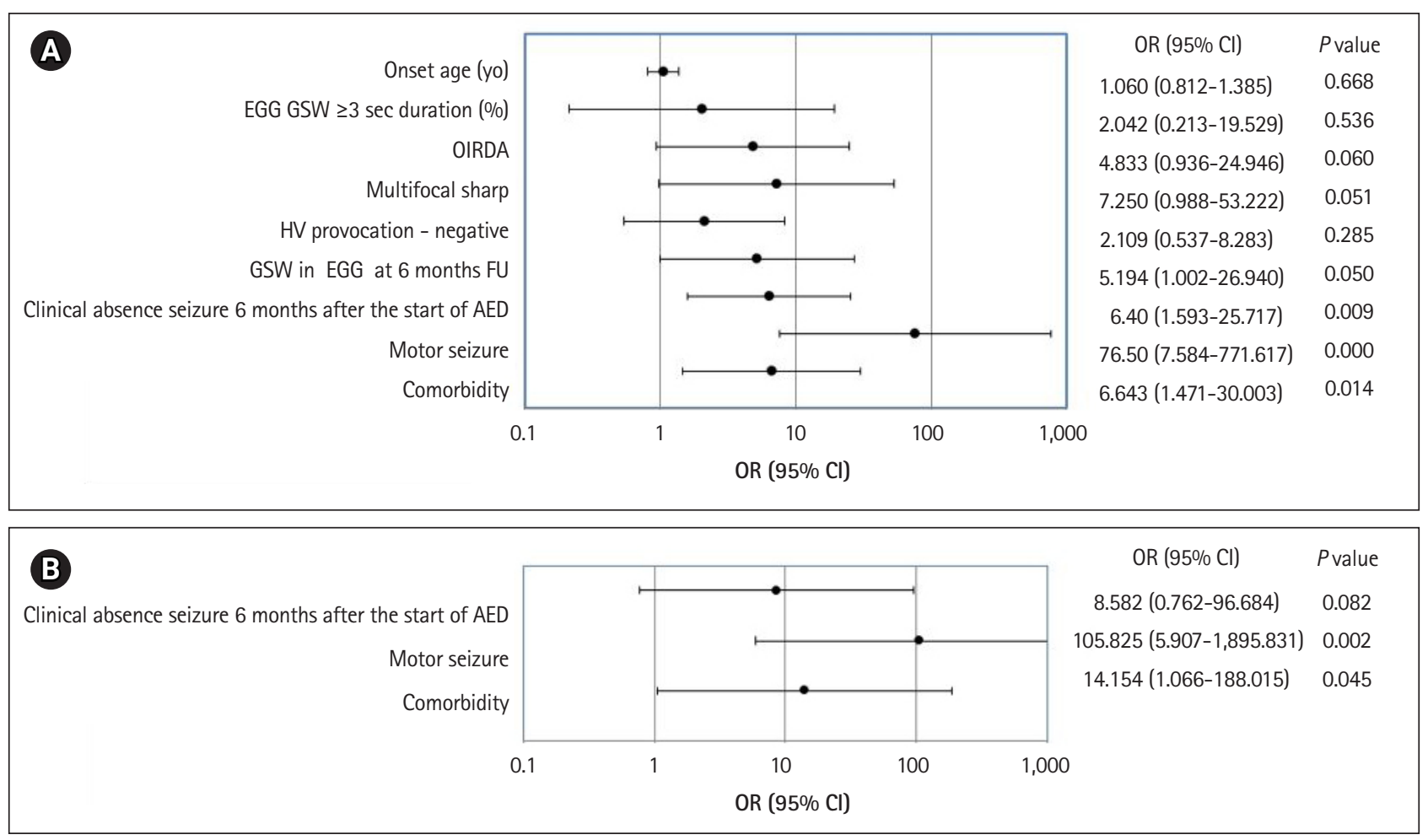

Fig. 1. Graphic presentation of the univariate and multivariate analysis for the poor prognostic factors in patients with childhood absence epilepsy. (A) Univariate analysis. (B) Multivariate analysis. OR, odds ratio; $\mathrm{Cl}$, confidence interval; yo, years old; $\mathrm{EEG}$, electroencephalogram; GSW, generalized spike-and-wave complexes; OIRDA, occipital intermittent rhythmic delta activity; HV, hyperventilation; FU, follow-up; AED, antiepileptic drug.

sponse to AEDs [2,3]. Unfavorable prognostic factors include late onset age, nonpyknoleptic absence seizure patterns, later development of myoclonic attacks or GTCs, atypical EEG features, psychiatric comorbidities, and side effects of AEDs [2-4,6,8,10,11].

In an earlier study, of 39 adult patients with typical absence seizures from 10 years of age, $12 \%$ were diagnosed with JAE and $7 \%$ with JME [12]. There is however a period of overlap between CAE and JME. In a retrospective study, $18 \%$ of patients diagnosed with JME evolved from CAE [13]. Linkage to chromosome 1 has also been reported in patients with absence seizures from childhood that were later diagnosed with JME [6,14]. In addition, several chromosomal loci have been identified in families of patients with absence seizures from childhood $[6,15]$. Recently, several epilepsy genes have been found in idiopathic generalized epilepsies with unclear family history and a genetic mutation in gamma-aminobutyric acid receptor alpha- 1 has been found in CAE and JME $[6,16]$.

In this study, only monotherapy was started at the time of initiation and more than $50 \%$ of patients started treatment with ETX, considering the effects and side effects. This study shows that the presence of clinical absence seizures after 6 months of taking AEDs, motor seizures, and comorbidities are the most important predictive factors for an unfavorable prognosis. Generally, GTC or myoclonic jerks are not suitable for CAE diagnosis. However, in this journal, we tried to evaluate whether the prognosis is worse if patients initially diagnosed with typical CAE had even once motor seizure. In patients initially diagnosed with typical CAE, those with motor seizure progressing to JAE, JME showed the most unfavorable prognosis.

In this study, neuropsychological tests were performed at the time of the first diagnosis to check for neurodevelopment status and unknown comorbidity such as $\mathrm{ADHD}$ and attention deficits. The prognosis was also unfavorable when there was comorbidity $[10,11]$.

The neuropsychological test follow-up was not performed periodically after diagnosis in this study. But it may be necessary to check psychosocial outcome whether the learning difficulty is affected after diagnosis and treatment by neuropsychological test.

The absence of a clinical seizure within 6 months of taking AEDs can be considered as a good response to treatment. This study is in line with earlier reports showing similar results [2$4,8,10,11]$. EEG abnormalities after 6 months of therapy with AEDs was not identified as a poor prognostic factor in this study, as the statistical tests did not reveal significance. However, as in 
previous studies, the prognosis was five times worse when GSW complexes were observed on EEG 6 months after the administration of AEDs [2]. An EEG follow-up within 6 months to predict the prognosis would thus seem warranted.

Previous studies have shown a good prognosis with OIRDA and a poor prognosis with EEG polyspikes, but none of these two EEG abnormalities showed a significant correlation with prognosis in this study $[2-4,8,10,11]$. We assumed that the prognosis would be worse if the duration of visible GSW complexes was long or if they were not easily provokated by hyperventilation, but our analysis did not yield statistical significance.

This study is limited by its retrospective analysis approach as well as by its small sample size and single-center design. In addition, the lack of long-term follow-up observations has limited our assessment of epilepsy syndromes. Further studies are needed to investigate the relationships between prognostic factors and evolvement into other epilepsy syndromes, as well as potential relationships with genetic mutations.

In conclusion, our study suggests that in cases of clinical seizures even after 6 months of AED treatment, motor seizures, or comorbidities, early intervention should be considered. A prospective or randomized controlled clinical trial in the future might be able to provide more detailed results.

\section{Conflicts of interest}

No potential conflicts of interest relevant to this article was reported.

\section{ORCID}

So Young Kang, https://orcid.org/0000-0002-6079-9497

Hoon-Chul Kang, https://orcid.org/0000-0002-3659-8847

Chung Mo Koo, https://orcid.org/0000-0003-1434-6988

\section{References}

1. Scheffer IE, Berkovic S, Capovilla G, Connolly MB, French J, Guilhoto L, et al. ILAE classification of the epilepsies: position paper of the ILAE commission for classification and terminology. Epilepsia 2017;58:512-21.

2. Kim HR, Kim GH, Eun SH, Eun BL, Byeon JH. Therapeutic outcomes and prognostic factors in childhood absence epilepsy. J Clin Neurol 2016;12:160-5.

3. Grosso S, Galimberti D, Vezzosi P, Farnetani M, Di Bartolo RM,
Bazzotti S, et al. Childhood absence epilepsy: evolution and prognostic factors. Epilepsia 2005;46:1796-801.

4. Trinka E, Baumgartner S, Unterberger I, Unterrainer J, Luef G, Haberlandt E, et al. Long-term prognosis for childhood and juvenile absence epilepsy.J Neurol 2004;251:1235-41.

5. Commission on Classification and Terminology of the International League Against Epilepsy. Proposal for revised classification of epilepsies and epileptic syndromes. Epilepsia 1989; 30:389-99.

6. Bureau M. Epileptic syndromes in infancy, childhood and adolescence. 5th ed. Montrouge: John Libbey Eurotext; 2012. p. 600.

7. Glauser TA, Cnaan A, Shinnar S, Hirtz DG, Dlugos D, Masur D, et al. Ethosuximide, valproic acid, and lamotrigine in childhood absence epilepsy. N Engl J Med 2010;362:790-9.

8. Wirrell EC. Natural history of absence epilepsy in children. Can J Neurol Sci 2003;30:184-8.

9. Glauser TA, Cnaan A, Shinnar S, Hirtz DG, Dlugos D, Masur D, et al. Ethosuximide, valproic acid, and lamotrigine in childhood absence epilepsy: initial monotherapy outcomes at 12 months. Epilepsia 2013;54:141-55.

10. Masur D, Shinnar S, Cnaan A, Shinnar RC, Clark P, Wang J, et al. Pretreatment cognitive deficits and treatment effects on attention in childhood absence epilepsy. Neurology 2013;81: 1572-80.

11. Gomez-Ibanez A, McLachlan RS, Mirsattari SM, Diosy DC, Burneo JG. Prognostic factors in patients with refractory idiopathic generalized epilepsy. Epilepsy Res 2017;130:69-73.

12. Agathonikou A, Giannakodimos S, Ahmed-Saroqi I, Koutroumanidis M, Sanders S, Panyiotopoulos CP. Idiopathic generalised epilepsies in adults with onset of typical absences after the age of 10 years. Epilepsia 1997;38(Suppl 3):224-5.

13. Martinez-Juarez IE, Alonso ME, Medina MT, Duron RM, Bailey JN, Lopez-Ruiz M, et al. Juvenile myoclonic epilepsy subsyndromes: family studies and long-term follow-up. Brain 2006;129(Pt 5):1269-80.

14. Delgado-Escueta AV, Medina MT, Serratosa JM, Castroviejo IP, Gee MN, Weissbecker K, et al. Mapping and positional cloning of common idiopathic generalized epilepsies: juvenile myoclonus epilepsy and childhood absence epilepsy. Adv Neurol 1999;79:351-74.

15. Weber YG, Lerche H. Genetic mechanisms in idiopathic epilepsies. Dev Med Child Neurol 2008;50:648-54.

16. Wang J, Lin ZJ, Liu L, Xu HQ, Shi YW, Yi YH, et al. Epilepsy-associated genes. Seizure 2017;44:11-20. 